
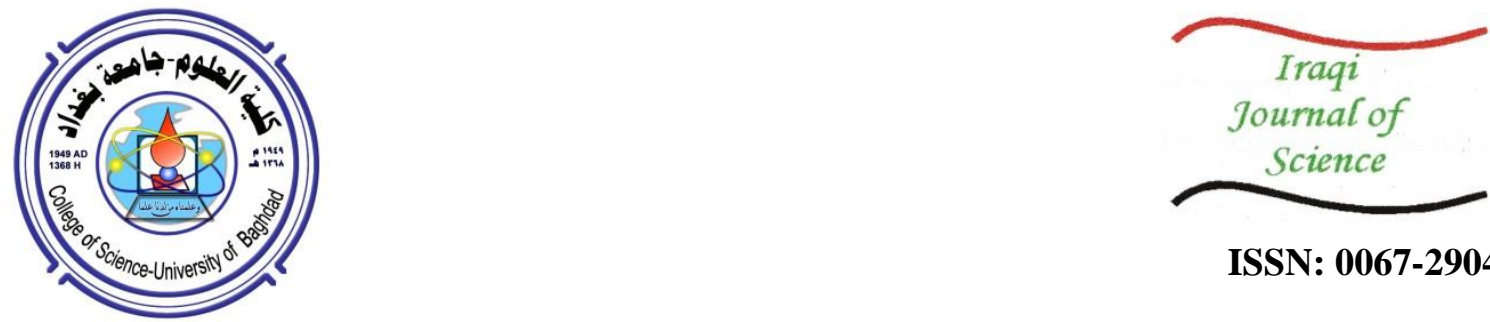

ISSN: 0067-2904

\title{
On Centralizers of 2-torsion Free Semiprime Gamma Rings
}

\author{
Abdulkareem T. Mutlak, Abulrahman H. Majeed* \\ Department of Mathematics, College of Science, University of Baghdad, Baghdad, Iraq
}

Received: 29/7/2020 Accepted: 23/9/2020

\begin{abstract}
In this paper, we prove that; Let $\mathrm{M}$ be a 2-torsion free semiprime $\Gamma-$ ring which satisfies the condition $x \alpha y \beta z=x \beta y \alpha z$ for all $x, y, z \in M$ and $\alpha, \beta \in \Gamma$. Consider that $T: M \rightarrow M$ as an additive mapping such that $2 T(x \alpha x)=T(x) \alpha x+x \alpha T(x)$ holds for all $x \in M$ and $\alpha \in \Gamma$, then $\mathrm{T}$ is a left and right centralizer.
\end{abstract}

Keywords: $\Gamma-$ ring , Prime $\Gamma-$ ring, Semiprime $\Gamma-$ ring, left centralizer, right centralizer, Jordan centralizer.

\section{حول تمركزات لحلقات كاما شبه الاولية طليقة الالتواء من النمط -2}

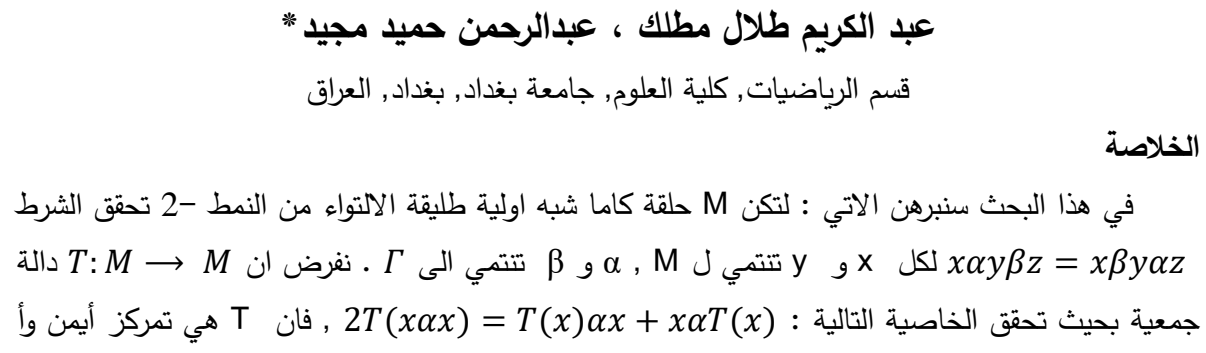

\section{Introduction}

An extensive generalized concept of classical rings was presented by the gamma ring theory. Bernes [1], Luh [2] and Kyuno [3] studied the structure of gamma rings and obtained various generalizations of corresponding parts in the ring theory.

Let $M$ and $\Gamma$ be additive abelian groups, if there exists a mapping $(x, \alpha, y) \rightarrow x \alpha y$ of $M \times \Gamma \times$ $M \rightarrow M$ which satisfies the conditions:

i. $x \alpha y \in M$.

ii. $(x+y) \alpha z=x \alpha z+y \alpha z, \quad x(\alpha+\beta) y=x \alpha y+x \beta y$ and $x \alpha(y+z)=x \alpha y+x \alpha z$.

iii. $(x \alpha y) \beta z=x \alpha(y \beta z)$ for all $x, y, z \in M$ and $\alpha, \beta \in \Gamma$.

Then, $\mathrm{M}$ is called a $\Gamma-\operatorname{ring}[1]$. A $\Gamma-\operatorname{ring} \mathrm{M}$ is called prime if $a \Gamma M \Gamma b=0$ implies $a=0$ or $b=0$ where $a, b \in M$. Also, $\mathrm{M}$ is called semiprime if $a \Gamma M \Gamma a=0$ implies $a=0$ where $a \in M$ [5]. Moreover, $\mathrm{M}$ is called 2-torsion free if $2 x=0$ implies $x=0$.

An additive map $T: M \rightarrow M$ is called a left (right) centralizer if $T(x \alpha y)=T(x) \alpha y(T(x \alpha y)=$ $x \alpha T(y))$ holds for all $x, y \in M$ and $\alpha \in \Gamma$. A centralizer is an additive mapping which is both a left and right centralizer [5].

Let $\mathrm{M}$ be a $\Gamma-\operatorname{ring}$, then $[x, y]_{\alpha}=x \alpha y-y \alpha x$ is known as commutator of $\mathrm{x}$ and $\mathrm{y}$ with respect to $\alpha$, where, $x, y \in M$ and $\alpha \in \Gamma$. In addition, the basic commutator identities are shown below [5]:

i. $\quad[x \alpha y, z]_{\beta}=[x, z]_{\beta} \alpha y+x[\alpha, \beta]_{z} y+x \alpha[y, z]_{\beta}$. 
ii. $\quad[x, y \alpha z]_{\beta}=[x, y]_{\beta} \alpha z+y[\alpha, \beta]_{x} z+y \alpha[x, z]_{\beta}$.

Now, we consider the following assumption, $x \alpha y \beta z=x \beta y \alpha z$ for all $x, y, z \in M$ and $\alpha, \beta \in \Gamma$

(A)

According to this assumption, the above two identities are reduced to:

i. $\quad[x \alpha y, z]_{\beta}=[x, z]_{\beta} \alpha y+x \alpha[y, z]_{\beta}$.

ii. $\quad[x, y \alpha z]_{\beta}=[x, y]_{\beta} \alpha z+y \alpha[x, z]_{\beta}$.

Hoque and Paul [5] proved that every Jordan centralizer of 2-torsion free semiprime $\Gamma-$ ring is centralizer. Many researchers have proved results on 2-torsion free semiprime $\Gamma$ - ring centralizer. In addition, many researchers worked on centralizer of prime and semiprime ring $[6,7]$.

Throughout this paper, we use condition (A) and assume that $T: M \rightarrow M$ is an additive mapping which satisfy the following condition:

$2 T(x \alpha x)=T(x) \alpha x+x \alpha T(x)$ for all $x \in M$ and $\alpha \in \Gamma$

\section{Results}

First, we need to prove some lemmas as in the following.

Lemma2.1: Let $M$ be a $\Gamma$-ring that has an identity element, $T: M \rightarrow M$ is a left (right) centralizer if and only if there exists $a \in M$ and $\alpha \in \Gamma$ such that $T(x)=a \alpha x(T(x)=x \alpha a)$ for all $x \in M$.

Proof: $\Leftarrow$ ) by assumption we have, $T(x)=a \alpha x$ for some $a \in M$ and $\alpha \in \Gamma$ and all $x \in M$.

$T(x \beta y)=a \alpha x \beta y=T(x) \beta y$ is a left centralizer for all $x, y \in M$ and $\alpha, \beta \in \Gamma$.

By a similar way, we can obtain that if $\mathrm{T}$ is satisfying that $T(x)=x \alpha a$ then we get $\mathrm{T}$ and is a right centralizer.

$\Rightarrow$ ) If $T(x \alpha y)=T(x) \alpha y$ for all $x, y \in M$ and $\alpha \in \Gamma$, then $T(1 \alpha x)=T(1) \alpha x$.

If we take $T(1)=a$ then we obtain, $T(x)=T(1 \alpha x)=a \alpha x$ for all $a, x \in M$ and $\alpha \in \Gamma$.

By a similar way, we can prove whether $\mathrm{T}$ is a right centralizer $T(x)=T(1 \alpha x)=x \alpha T(1)=x \alpha a$.

Lemma2.2: - Let $M$ be 2-torsion free semiprime $\Gamma-\operatorname{ring}$ satisfying condition (A) and $T: M \rightarrow M$ satisfying condition (B), then $8 T(x \alpha y \beta x)=T(x) \alpha(x \beta y+3 y \beta x)+(y \beta x+3 x \beta y) \alpha T(x)+$ $2 x \alpha T(y) \beta x-x \alpha x \beta T(y)-T(y) \beta x \alpha x$ where, $x, y \in M$ and $\alpha, \beta \in \Gamma$.

Proof: By replacing $\mathrm{x}$ in equation (B) by $x+y$ we get,

$$
2 T((x+y) \alpha(x+y))=T(x+y) \alpha(x+y)+(x+y) \alpha T(x+y)
$$

$2 T(x \alpha x)+2 T(x \alpha y)+2 T(y \alpha x)+2 T(y \alpha y)=T(x) \alpha x+T(x) \alpha y+T(y) \alpha x+T(y) \alpha y+$ $x \alpha T(x)+x \alpha T(y)+y \alpha T(x)+y \alpha T(y)$. Then, for all $x, y \in M$ and $\alpha \in \Gamma$ we obtain,

$$
2 T(x \alpha y+y \alpha x)=T(x) \alpha y+x \alpha T(y)+T(y) \alpha x+y \alpha T(x)
$$

Now, replacing y in equation (1) by $2(x \beta y+y \beta x)$ and then using equation (1) implies,

$4 T(x \alpha(x \beta y+y \beta x)+(x \beta y+y \beta x) \alpha x)$

$$
\begin{aligned}
= & 2 T(x) \alpha(x \beta y+y \beta x)+2 x \alpha T(x \beta y+y \beta x)+2 T(x \beta y+y \beta x) \alpha x \\
& +2(x \beta y+y \beta x) \alpha T(x) \\
=2 T(x) \alpha(x \beta y & +y \beta x)+x \alpha T(x) \beta y+x \alpha x \beta T(y)+x \alpha T(y) \beta x+x \alpha y \beta T(x)+T(x) \beta y \alpha x \\
& +x \beta T(y) \alpha x+T(y) \beta x \alpha x+y \beta T(x) \alpha x+2(x \beta y+y \beta x) \alpha T(x) .
\end{aligned}
$$

By simplifying the above equation, we obtain,

$$
\begin{array}{r}
4 T(x \alpha(x \beta y+y \beta x)+(x \beta y+y \beta x) \alpha x)=T(x) \alpha(2 x \beta y+3 y \beta x)+(3 x \beta y+2 y \beta x) \alpha T(x)+ \\
x \alpha T(x) \beta y+y \beta T(x) \alpha x+2 x \alpha T(y) \beta x+x \alpha x \beta T(y)+T(y) \beta x \alpha x
\end{array}
$$

On the other hand, by using equation (1) and equation (B) we get,

$$
\begin{aligned}
4 T(x \alpha(x \beta y+ & y \beta x)+(x \beta y+y \beta x) \alpha x)=4 T(x \alpha x \beta y)+4 T(x \alpha y \beta x)+4 T(x \beta y \alpha x)+4 T(y \beta x \alpha x) \\
& =4 T(x \alpha x \beta y)+4 T(y \beta x \alpha x)+8 T(x \alpha y \beta x) \\
& =2 T(2 x \alpha x \beta y+2 y \beta x \alpha x)+8 T(x \alpha y \beta x) \\
& =2 T(x \alpha x) \beta y+2 x \alpha x \beta T(y)+2 T(y) \beta x \alpha x+2 y \beta T(x \alpha x)+8 T(x \alpha y \beta x) .
\end{aligned}
$$

Hence, for all $x, y \in M$ and $\alpha, \beta \in \Gamma$ we have,

$4 T(x \alpha(x \beta y+y \beta x)+(x \beta y+y \beta x) \alpha x)=T(x) \alpha x \beta y+y \beta x \alpha T(x)+x \alpha T(x) \beta y+y \beta T(x) \alpha x+$ $2 x \alpha x \beta T(y)+2 T(y) \beta x \alpha x++8 T(x \alpha y \beta x)$

By comparing equation (2) with equation (3) we get,

$$
\begin{gathered}
8 T(x \alpha y \beta x)=T(x) \alpha(x \beta y+3 y \beta x)+(y \beta x+3 x \beta y) \alpha T(x)+2 x \alpha T(y) \beta x-x \alpha x \beta T(y) \\
-T(y) \beta x \alpha x \text { for all } x, y \in M \text { and } \alpha, \beta \in \Gamma
\end{gathered}
$$

Lemma2.3: - Let $M$ be 2-torsion free semiprime $\Gamma-\operatorname{ring}$ satisfying condition (A) and $T: M \rightarrow M$ satisfying condition (B), then for all $x, y \in M$ and $\alpha, \beta \in \Gamma$ we have, 
$T(x) \alpha(x \alpha y \beta x-2 y \beta x \alpha x-2 x \alpha x \beta y)+(x \alpha y \beta x-2 x \alpha x \beta y-2 y \beta x \alpha x) \alpha T(x)+x \beta T(x) \alpha(x \beta y+$ $y \beta x)+(x \beta y+y \beta x) \alpha T(x) \beta x+x \beta x \alpha T(x) \beta y+y \beta T(x) \alpha x \beta x=0$

Proof: By using equation (1) with replacing y by $8 x \alpha y \beta x$ and then using equation (4) we get, $16 T(x \alpha x \alpha y \beta x+x \alpha y \beta x \alpha x)=8 T(x) \alpha x \alpha y \beta x+8 x \alpha T(x \alpha y \beta x)+8 T(x \alpha y \beta x) \alpha x+8 x \alpha y \beta x \alpha T(x)$ $=8 T(x) \alpha x \alpha y \beta x+x \alpha T(x) \alpha(x \beta y+3 y \beta x)+(x \alpha y \beta x+3 x \alpha x \beta y) \alpha T(x)$ $+2 x \alpha x \alpha T(y) \beta x-x \alpha x \alpha x \beta T(y)-x \alpha T(y) \beta x \alpha x+T(x) \alpha(x \beta y \alpha x+y \beta x \alpha x)$ $+(y \beta x+3 x \beta y) \alpha T(x) \alpha x+2 x \alpha T(y) \beta x \alpha x-x \alpha x \beta T(y) \alpha x-T(y) \beta \alpha x \alpha x$ $+8 x \alpha y \beta x T(x)=0$.

Therefore, for all $x, y \in M$ and $\alpha, \beta \in \Gamma$ we can get,

$16 T(x \alpha x \alpha y \beta x+x \alpha y \beta x \alpha x)=T(x) \alpha(9 x \alpha y \beta x+3 y \beta x \alpha x)+(9 x \alpha y \beta x+3 x \alpha x \beta y) \alpha T(x)+$ $x \alpha T(x) \alpha(x \beta y+3 y \beta x)+(y \beta x+3 x \beta y) \alpha T(x) \alpha x+x \alpha x \alpha T(y) \beta x+x \alpha T(y) \beta x \alpha x-$ $T(y) \beta x \alpha x \alpha x-x \alpha x \alpha x \beta T(y)$

We can obtain the other hand by using equation (4) and then, after collecting some terms, using equation (1), as follows:

$$
\begin{aligned}
& 16 T(x \alpha x \alpha y \beta x+x \beta y \alpha x \alpha x)=16(x \alpha(x \alpha y) \beta x)+16 T(x \alpha(y \alpha x) \beta x) \\
& =2 T(x) \alpha(x \beta x \alpha y+3 x \alpha y \beta x)+2(x \alpha y \beta x+3 x \beta x \alpha y) \alpha T(x)+4 x \alpha T(x \alpha y) \beta x-2 x \alpha x \beta T(x \alpha y) \\
& -2 T(x \alpha y) \beta x \alpha x+2 T(x) \alpha(x \alpha y \beta x+3 y \beta x \alpha x)+2(y \beta x \alpha x+3 x \alpha y \beta x) \alpha T(x) \\
& +4 x \alpha T(y \alpha x) \beta x-2 x \alpha x \beta T(y \alpha x)-2 T(y \alpha x) \beta x \alpha x \text {. } \\
& =T(x) \alpha(2 x \alpha x \beta y+6 y \beta x \alpha x+8 x \alpha y \beta x)+(8 x \alpha y \beta x+2 y \beta x \alpha x+6 x \alpha x \beta y) \alpha T(x) \\
& +4 x \alpha T(x \beta y+y \beta x) \alpha x-2 x \alpha x \alpha T(x \beta y+y \beta x)-2 T(x \beta y+y \beta x) \alpha x \alpha x \text {. } \\
& = \\
& T(x) \alpha(2 x \alpha x \beta y+6 y \beta x \alpha x+8 x \alpha y \beta x)+(8 x \alpha y \beta x+2 y \beta x \alpha x+6 x \alpha x \beta y) \alpha T(x)+ \\
& 2 x \alpha T(x) \beta y \alpha x+2 x \alpha x \beta T(y) \alpha x+2 x \alpha T(y) \beta x \alpha x+2 x \alpha y \beta T(x) \alpha x-x \alpha x \alpha T(x) \beta y- \\
& x \alpha x \alpha x \beta T(y)-x \alpha x \beta T(y) \alpha x-x \alpha x \beta y \alpha T(x)-x \alpha T(y) \beta x \alpha x-T(y) \beta x \alpha x \alpha x-y \beta T(x) \alpha x \alpha x \text {. } \\
& \text { Hence, for all } x, y \in M \text { and } \alpha, \beta \in \Gamma \text { we have, } \\
& 16 T(x \alpha x \alpha y \beta x+x \beta y \alpha x \alpha x) \\
& =T(x) \alpha(2 x \alpha x \beta y+5 y \beta x \alpha x+8 x \alpha y \beta x)+(2 y \beta x \alpha x+5 x \alpha x \beta y+8 x \alpha y \beta x) \alpha T(x) \\
& +2 x \alpha T(x) \beta y \alpha x+2 x \alpha y \beta T(x) \alpha x+x \alpha x \beta T(y) \alpha x+x \alpha T(y) \beta x \alpha x-x \alpha x \alpha T(x) \beta y \\
& -y \beta T(x) \alpha x \alpha x-x \alpha x \alpha x \beta T(y) \\
& -T(y) \beta x \alpha x \alpha x
\end{aligned}
$$

By comparing equation (6) with equation (7), we obtain equation (5) which is the result.

Lemma2.4: - Let $M$ be 2-torsion free Semiprime $\Gamma$ - ring satisfying condition (A) and $T: M \rightarrow M$ satisfying condition (B), then $[T(x), x \alpha x]_{\alpha}=0$, for all $x \in M$ and $\alpha \in \Gamma$.

Proof: By putting $y \alpha x$ instead of $y$ in equation (5) we obtain,

$T(x) \alpha(x \alpha y \alpha x \beta x-2 y \alpha x \beta x \alpha x-2 x \alpha x \beta y \alpha x)+(x \alpha y \alpha x \beta x-2 x \alpha x \beta y \alpha x-2 y \alpha x \beta x \alpha x) \alpha T(x)$

$+x \beta T(x) \alpha(x \beta y \alpha x+y \alpha x \beta x)+(x \beta y \alpha x+y \alpha x \beta x) \alpha T(x) \beta x+x \beta x \alpha T(x) \beta y \alpha x$

$+y \alpha x \beta T(x) \alpha x \beta x=0$

Right multiplication of equation (5) by $\mathrm{x}$ gives for all $x, y \in M$ and $\alpha, \beta \in \Gamma$ the equation, $T(x) \alpha(x \alpha y \alpha x \beta x-2 y \alpha x \beta x \alpha x-2 x \alpha x \beta y \alpha x)+(x \alpha y \beta x-2 x \alpha x \beta y-2 y \beta x \alpha x) \alpha T(x) \alpha x$ $+x \beta T(x) \alpha(x \beta y \alpha x+y \alpha x \beta x)+(x \beta y-2 y \beta x \alpha x) \alpha T(x) \beta x \alpha x+x \beta x \alpha T(x) \beta y \alpha x$ $+y \beta T(x) \alpha x \beta x \alpha x=0$

Now, by subtracting equation (9) from equation (8) we get,

$(x \alpha y \alpha x \beta x-2 x \alpha x \beta y \alpha x-2 y \alpha x \beta x \alpha x) \alpha T(x)+(x \beta y \alpha x+y \alpha x \beta x) \alpha T(x) \beta x+y \alpha x \beta T(x) \alpha x \beta x$ $-(x \alpha y \beta x-2 x \alpha x \beta y-2 y \beta x \alpha x) \alpha T(x) \alpha x-(x \beta y+y \beta x) \alpha T(x) \beta x \alpha x$ $-(y \beta T(x) \alpha x \beta x \alpha x=0$ for all $x, y \in M$ and $\alpha, \beta \in \Gamma$.

$\mathrm{x} \alpha y \beta x \alpha[x, T(x)]_{\alpha}+2 x \alpha x \beta y \alpha[T(x), x]_{\alpha}+2 y \beta x \alpha x \alpha[T(x), x]_{\alpha}+x \beta y \alpha[x, T(x)]_{\alpha} \beta x+$ $y \beta x \alpha[x, T(x)]_{\alpha} \beta x+y \beta[x, T(x)]_{\alpha} \beta x \alpha x=0$ for all $x, y \in M$ and $\alpha, \beta \in \Gamma$.

Let $\alpha=\beta$ and by collecting the first and the fourth terms together we get, for all $x, y \in M$ and $\alpha \in$ $\Gamma$, the following equation:

$x \alpha y \alpha[x \alpha x, T(x)]_{\alpha}+2 x \alpha x \alpha y \alpha[T(x), x]_{\alpha}+2 y \alpha x \alpha x \alpha[T(x), x]_{\alpha}+y \alpha x \alpha[x, T(x)]_{\alpha} \alpha x+$ $y \alpha[x, T(x)]_{\alpha} \alpha x \alpha x=0$

Substituting $T(x) \alpha y$ for $y$ in the equation (10) implies, $x \alpha T(x) \alpha y \alpha[x \alpha x, T(x)]_{\alpha}+2 x \alpha x \alpha T(x) \alpha y \alpha[T(x), x]_{\alpha}+2 T(x) \alpha y \alpha x \alpha x \alpha[T(x), x]_{\alpha}+$ $T(x) \alpha y \alpha x \alpha[x, T(x)]_{\alpha} \alpha x+T(x) \alpha y \alpha[x, T(x)]_{\alpha} \alpha x \alpha x=0$ 
Left multiplication of equation (10) by $T(x)$ leads to,

$T(x) \alpha x \alpha y \alpha[x \alpha x, T(x)]_{\alpha}+2 T(x) \alpha x \alpha x \alpha y \alpha[T(x), x]_{\alpha}+2 T(x) \alpha y \alpha x \alpha x \alpha[T(x), x]_{\alpha}+$ $T(x) \alpha y \alpha x \alpha[x, T(x)]_{\alpha} \alpha x+T(x) \alpha y \alpha[x, T(x)]_{\alpha} \alpha x \alpha x=0$

By subtracting equation (12) from equation (11) we obtain,

$x \alpha T(x) \alpha y \alpha[x \alpha x, T(x)]_{\alpha}+2 x \alpha x \alpha T(x) \alpha y \alpha[T(x), x]_{\alpha}-T(x) \alpha x \alpha y \alpha[x \alpha x, T(x)]_{\alpha}-$

$2 T(x) \alpha x \alpha x \alpha y \alpha[T(x), x]_{\alpha}=0$ for all $x, y \in M$ and $\alpha \in \Gamma$.

Now, for all $x, y \in M$ and $\alpha \in \Gamma$ we have,

$[T(x), x]_{\alpha} \alpha y \alpha[T(x), x \alpha x]_{\alpha}-2[T(x), x \alpha x]_{\alpha} \alpha y \alpha[T(x), x]_{\alpha}=0$

By putting $y \alpha[T(x), x]_{\alpha} \alpha z$ instead of $y$ in equation (13) we get for all $x, y, z \in M$ and $\alpha \in \Gamma$,

$[T(x), x]_{\alpha} \alpha y \alpha[T(x), x]_{\alpha} \alpha z \alpha[T(x), x \alpha x]_{\alpha}-2[T(x), x \alpha x]_{\alpha} \alpha y \alpha[T(x), x]_{\alpha} \alpha z \alpha[T(x), x]_{\alpha}=0$..

Left multiplication of equation (13) by $[T(x), x]_{\alpha} \alpha y$ implies,

$$
\begin{gathered}
{[T(x), x]_{\alpha} \alpha y \alpha[T(x), x]_{\alpha} \alpha z \alpha[T(x), x \alpha x]_{\alpha}+[T(x), x]_{\alpha} \alpha y \alpha[-2 T(x), x \alpha x]_{\alpha} \alpha z \alpha[T(x), x]_{\alpha}} \\
=0
\end{gathered}
$$

By subtracting equation (15) from equation (14) for all $x, y, z \in M$ and $\alpha \in \Gamma$ we have,

$$
\begin{gathered}
\left([T(x), x]_{\alpha} \alpha y \alpha[-2 T(x), x \alpha x]_{\alpha}+[2 T(x), x \alpha x]_{\alpha} \alpha y \alpha[T(x), x]_{\alpha}\right) \\
\alpha z \alpha[T(x), x]_{\alpha}=0
\end{gathered}
$$

Let, in equation (16), $z$ be $z \alpha[-2 T(x), x \alpha x]_{\alpha} \alpha y$, then we obtain for all $x, y, z \in M$ and $\alpha \in \Gamma$,

$\left([T(x), x]_{\alpha} \alpha y \alpha[-2 T(x), x \alpha x]_{\alpha}+\right.$

$\left.[2 T(x), x \alpha x]_{\alpha} \alpha y \alpha[T(x), x]_{\alpha}\right) \alpha z \alpha[-2 T(x), x \alpha x]_{\alpha} \alpha y \alpha[T(x), x]_{\alpha}=0$

Right multiplication of equation (16) by $y \alpha[-2 T(x), x \alpha x]_{\alpha}$ gives,

$\left([T(x), x]_{\alpha} \alpha y \alpha[-2 T(x), x \alpha x]_{\alpha}+\right.$

$\left.[2 T(x), x \alpha x]_{\alpha} \alpha y \alpha[T(x), x]_{\alpha}\right) \alpha z \alpha[T(x), x]_{\alpha} \alpha y \alpha[-2 T(x), x \alpha x]_{\alpha}=0$

By subtracting equation (17) from equation (18) we obtain,

$\left([T(x), x]_{\alpha} \alpha y \alpha[-2 T(x), x \alpha x]_{\alpha}+\right.$

$\left.[2 T(x), x \alpha x]_{\alpha} \alpha y \alpha[T(x), x]_{\alpha}\right) \alpha z \alpha\left([T(x), x]_{\alpha} \alpha y \alpha[-2 T(x), x \alpha x]_{\alpha}+\right.$

$\left.[2 T(x), x \alpha x]_{\alpha} \alpha y \alpha[T(x), x]_{\alpha}\right)=0$ for all $x, y, z \in M$ and $\alpha \in \Gamma$.

$[T(x), x]_{\alpha} \alpha y \alpha[2 T(x), x \alpha x]_{\alpha}=[2 T(x), x \alpha x]_{\alpha} \alpha y \alpha[T(x), x]_{\alpha}$

Combining equation (13) with equation (19) leads to,

$[T(x), x]_{\alpha} \alpha y \alpha\left([T(x), x \alpha x]_{\alpha}-2[T(x), x \alpha x]_{\alpha}\right)$ for all $x, y \in M$ and $\alpha \in \Gamma$.

This implies that $[T(x), x]_{\alpha} \alpha y \alpha[T(x), x \alpha x]_{\alpha}=0 \quad$ for all $\quad x, y \in M \quad$ and $\quad \alpha \in \Gamma$ ...(20)

By left multiplying equation (20) by $x$ we obtain,

$x \alpha[T(x), x]_{\alpha} \alpha y \alpha[T(x), x \alpha x]_{\alpha}=0$ for all $x, y \in M$ and $\alpha \in \Gamma$

By replacing $x \alpha y$ for $y$ in equation (21) we get,

$[T(x), x]_{\alpha} \alpha x \alpha y \alpha[T(x), x \alpha x]_{\alpha}=0$ for all $x, y \in M$ and $\alpha \in \Gamma$

We combine equation (22) with equation (23) and the result is,

$\left([T(x), x]_{\alpha} \alpha x+x \alpha[T(x), x]_{\alpha}\right) \alpha y \alpha[T(x), x \alpha x]_{\alpha}=0$ for all $x, y \in M$ and $\alpha \in \Gamma$.

$[T(x), x \alpha x]_{\alpha} \alpha y \alpha[T(x), x \alpha x]_{\alpha}=0$ for all $x, y \in M$ and $\alpha \in \Gamma$

By semiprimness, we have, $[T(x), x \alpha x]_{\alpha}=0$ for all $x \in M$ and $\alpha \in \Gamma$

Lemma 2.5: - Let $M$ be 2-torsion free Semiprime $\Gamma$-ring satisfying condition $(A)$ and $T: M \rightarrow M$ satisfying condition (B), then, $[T(x), x]_{\alpha} \alpha x \alpha x=0, x \alpha x \alpha[T(x), x]_{\alpha}=0$ and $x \alpha[T(x), x]_{\alpha} \alpha x=0$ for all $x \in M$ and $\alpha \in \Gamma$.

Proof: For Lemma 2.4, we have equation (24). By the substitution of $x+y$ for $x$ in equation (24) we obtain,

$$
\begin{aligned}
& {[T(x+y),(x+y) \alpha(x+y)]_{\alpha}} \\
& \quad=[T(x), x \alpha x+y \alpha y+x \alpha y+y \alpha x]_{\alpha}+[T(y), x \alpha x+y \alpha y+x \alpha y+y \alpha x]_{\alpha}
\end{aligned}
$$

Hence, for all $x, y \in M$ and $\alpha \in \Gamma$,

$[T(x), y \alpha y]_{\alpha}+[T(y), x \alpha x]_{\alpha}+[T(x), x \alpha y+y \alpha x]_{\alpha}+[T(y), x \alpha y+y \alpha x]_{\alpha}=0$.

Putting, in the above equation, $-x$ for $x$ implies for all $x, y \in M$ and $\alpha \in \Gamma$,

$[-T(x), y \alpha y]_{\alpha}+[T(y), x \alpha x]_{\alpha}+[-T(x),-x \alpha y-y \alpha x]_{\alpha}+[T(y),-x \alpha y-y \alpha x]_{\alpha}=0$

By comparing the above two equations we have,

$[T(x), x \alpha y+y \alpha x]_{\alpha}+[T(y), x \alpha x]_{\alpha}=0$ for all $x, y \in M$ and $\alpha \in \Gamma$

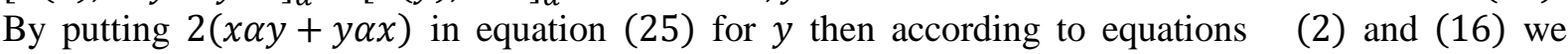
obtain, 


$$
\begin{gathered}
2[T(x), x \alpha(x \alpha y+y \alpha x)+(x \alpha y+y \alpha x)]_{\alpha}+[2 T(x \alpha y+y \alpha x), x \alpha x]_{\alpha}=0 \\
2[T(x), x \alpha x \alpha y+2 x \alpha y \alpha x+y \alpha x \alpha x]_{\alpha}+[T(x) \alpha y+x \alpha T(y)+T(y) \alpha x+y \alpha T(x), x \alpha x]_{\alpha}=0 \\
2[T(x), x \alpha x \alpha y]_{\alpha}+2[T(x), y \alpha x \alpha x]_{\alpha}+4[T(x), x \alpha y \alpha x]_{\alpha}+[T(x) y \alpha, x \alpha x]_{\alpha}+[x \alpha T(y), x \alpha x]_{\alpha} \\
+[T(y) \alpha x, x \alpha x]_{\alpha}+[y \alpha T(x), x \alpha x]_{\alpha}=0 \\
2 x \alpha x \alpha[T(x), y]_{\alpha}+2[T(x), y]_{\alpha} \alpha x \alpha x+4[T(x), x \alpha y \alpha x]_{\alpha}+T(x) \alpha[y, x \alpha x]_{\alpha}+x \alpha[T(y), x \alpha x]_{\alpha}+ \\
{[T(y), x \alpha x]_{\alpha} \alpha x+[y, x \alpha x]_{\alpha} \alpha T(x)=0}
\end{gathered}
$$

Thus, for all $x, y \in M$ and $\alpha \in \Gamma$ we have,

$2 x \alpha x \alpha[T(x), y]_{\alpha}+2[T(x), y]_{\alpha} \alpha x \alpha x+4[T(x), x \alpha y \alpha x]_{\alpha}+T(x) \alpha[y, x \alpha x]_{\alpha}+[y, x \alpha x]_{\alpha} \alpha T(x)$

$$
+x \alpha[T(y), x \alpha x]_{\alpha}+[T(y), x \alpha x]_{\alpha} \alpha x=0
$$

For $y=x$, equation (27) reduces to,

$$
x \alpha x \alpha[T(x), x]_{\alpha}+[T(x), x]_{\alpha} \alpha x \alpha x+2[T(x), x \alpha x \alpha x]_{\alpha}=0
$$

$x \alpha x \alpha[T(x), x]_{\alpha}+[T(x), x]_{\alpha} \alpha x \alpha x+2[T(x), x]_{\alpha} \alpha x \alpha x+2 x \alpha[T(x), x \alpha x]_{\alpha}=0$

Which gives, $\quad x \alpha x \alpha[T(x), x]_{\alpha}+3[T(x), x]_{\alpha} \alpha x \alpha x=0$ for all $x \in M$ and $\alpha \in \Gamma$

From equation (25) we get, $[T(x), x]_{\alpha} \alpha x+x \alpha[T(x), x]_{\alpha}=0$ for all $x \in M$ and $\alpha \in \Gamma$.

By right multiplication of the above relation by $x$ we get,

$$
[T(x), x]_{\alpha} \alpha x \alpha x+x \alpha[T(x), x]_{\alpha} \alpha x=0 \text { for all } x \in M \text { and } \alpha \in \Gamma
$$

Now, by left multiplication of the above relation by $x$ we have,

$$
x \alpha[T(x), x]_{\alpha} \alpha x+x \alpha x \alpha[T(x), x]_{\alpha}=0 \text { for all } x \in M \text { and } \alpha \in \Gamma
$$

Comparing equation (28) with equation (29) gives,

$$
[T(x), x]_{\alpha} \alpha x \alpha x=x \alpha x \alpha[T(x), x]_{\alpha} \text { for all } x \in M \text { and } \alpha \in \Gamma
$$

From equation (27) and equation (30) we obtain, $4 x \alpha x \alpha[T(x), x]_{\alpha}=0$ for all $x \in M$ and $\alpha \in \Gamma$, implies, $x \alpha x \alpha[T(x), x]_{\alpha}=0$ for all $x \in M$ and $\alpha \in \Gamma$

$$
[T(x), x]_{\alpha} \alpha x \alpha x=0 \text { for all } x \in M \text { and } \alpha \in \Gamma
$$

In addition, from equation (31) we have, $x \alpha[T(x), x]_{\alpha} \alpha x=0$ for all $x \in M$ and $\alpha \in \Gamma$

Lemma 2.6: Let $M$ be 2-torsion free semiprime $\Gamma$-ring satisfying condition (A)

and let $T: M \rightarrow M$ satisfying condition (B), then

$[T(x), x]_{\alpha} \alpha x=0$ and $x \alpha[T(x), x]_{\alpha}=0$ for all $x \in M$ and $\alpha \in \Gamma$.

Proof: From equation (25) we get, $[T(y), x \alpha x]_{\alpha}=-[T(x), x \alpha y+y \alpha x]_{\alpha}$.

Left multiplication of the above equation by $x$ gives, $x \alpha[T(y), x \alpha x]_{\alpha}=-x \alpha[T(x), x \alpha y+y \alpha x]_{\alpha}$.

Similarly, right multiplication by $x$ gives, $x \alpha[T(y), x \alpha x]_{\alpha}=-x \alpha[T(x), x \alpha y+y \alpha x]_{\alpha}$.

$[T(y), x \alpha x]_{\alpha} \alpha x=-[T(x), x \alpha y+y \alpha x]_{\alpha} \alpha x$ for all $x, y \in M$ and $\alpha \in \Gamma$.

Putting the above two equations in equation (26) gives,

$0=2 x \alpha x \alpha[T(x), y]_{\alpha}+2[T(x), y]_{\alpha} \alpha x \alpha x+4[T(x), x \alpha y \alpha x]_{\alpha}+T(x) \alpha[y, x \alpha x]_{\alpha}+[y, x \alpha x]_{\alpha} \alpha T(x)$

$-x \alpha[T(x), x \alpha y+y \alpha x]_{\alpha}-[T(x), x \alpha y+y \alpha x]_{\alpha} \alpha x$

$=2 x \alpha x \alpha[T(x), y]_{\alpha}+2[T(x), y]_{\alpha} \alpha x \alpha x+4[T(x), x]_{\alpha} \alpha y \alpha x+4 x \alpha[T(x), y]_{\alpha} \alpha x$

$+4 x \alpha y \alpha[T(x), x]_{\alpha}+T(x) \alpha[y, x \alpha x]_{\alpha}+[y, x \alpha x]_{\alpha} \alpha T(x)-x \alpha[T(x), x]_{\alpha} \alpha y$

$-x \alpha x \alpha[T(x), y]_{\alpha}-x \alpha y \alpha[T(x), x]_{\alpha}-x \alpha[T(x), y]_{\alpha} \alpha x-x \alpha[T(x), y]_{\alpha} \alpha x$

$-[T(x), x]_{\alpha} \alpha y \alpha x-y \alpha[T(x), x]_{\alpha} \alpha x-[T(x), y]_{\alpha} \alpha x \alpha x$.

Therefore, for all $x, y \in M$ and $\alpha \in \Gamma$,

$x \alpha x \alpha[T(x), y]_{\alpha}+[T(x), y]_{\alpha} \alpha x \alpha x+3[T(x), x]_{\alpha} \alpha y \alpha x+2 x \alpha[T(x), y]_{\alpha} \alpha x+3 x \alpha y \alpha[T(x), x]_{\alpha}+$

$T(x) \alpha[y, x \alpha x]_{\alpha}+[y, x \alpha x]_{\alpha} \alpha T(x)-x \alpha[T(x), x]_{\alpha} \alpha y-y \alpha[T(x), x]_{\alpha} \alpha x=0$

By the substitution of $y \alpha x$ for $y$ in equation (34) we get,

$x \alpha x \alpha[T(x), y \alpha x]_{\alpha}+[T(x), y \alpha x]_{\alpha} \alpha x \alpha x+3[T(x), x]_{\alpha} \alpha y \alpha x \alpha x+2 x \alpha[T(x), y \alpha x]_{\alpha} \alpha x+$

$3 x \alpha y \alpha x \alpha[T(x), x]_{\alpha}+T(x) \alpha[y \alpha x, x \alpha x]_{\alpha}+[y \alpha x, x \alpha x]_{\alpha} \alpha T(x)-x \alpha[T(x), x]_{\alpha} \alpha y \alpha x-$

$y \alpha x \alpha[T(x), x]_{\alpha} \alpha x=0$

$x \alpha x \alpha[T(x), y]_{\alpha} \alpha x+x \alpha x \alpha y \alpha[T(x), x]_{\alpha}+[T(x), y]_{\alpha} \alpha x \alpha x \alpha x+y \alpha[T(x), x]_{\alpha} \alpha x \alpha x+$

$3[T(x), x]_{\alpha} \alpha y \alpha x \alpha x+2 x \alpha[T(x), y]_{\alpha} \alpha x \alpha x+2 x \alpha y \alpha[T(x), x]_{\alpha} \alpha x+3 x \alpha y \alpha x \alpha[T(x), x]_{\alpha}+$

$T(x) \alpha[y, x \alpha x]_{\alpha} \alpha x+[y, x \alpha x]_{\alpha} \alpha x \alpha T(x)-x \alpha[T(x), x]_{\alpha} \alpha y \alpha x-y \alpha x \alpha[T(x), x]_{\alpha} \alpha x=0$

This reduces equation (32) and equation (33) to,

$x \alpha x \alpha[T(x), y]_{\alpha} \alpha x+x \alpha x \alpha y \alpha[T(x), x]_{\alpha}+[T(x), y]_{\alpha} \alpha x \alpha x \alpha x+3 x \alpha y \alpha x \alpha[T(x), x]_{\alpha}+$

$2 x \alpha[T(x), y]_{\alpha} \alpha x \alpha x+2 x \alpha y \alpha[T(x), x]_{\alpha} \alpha x+T(x) \alpha[y, x \alpha x]_{\alpha} \alpha x+[y, x \alpha x]_{\alpha} \alpha x \alpha T(x)-$

$x \alpha[T(x), x]_{\alpha} \alpha y \alpha x=0$ for all $x, y \in M$ and $\alpha \in \Gamma$

Right multiplication of equation (34) by $x$ gives, for all $x, y \in M$ and $\alpha \in \Gamma$ 
$x \alpha x \alpha[T(x), y]_{\alpha} \alpha x+[T(x), y]_{\alpha} \alpha x \alpha x \alpha x+3[T(x), x]_{\alpha} \alpha y \alpha x \alpha x+3 x \alpha y \alpha[T(x), x]_{\alpha} \alpha x+$ $2 x \alpha[T(x), y]_{\alpha} \alpha x \alpha x+T(x) \alpha[y, x \alpha x]_{\alpha} \alpha x+[y, x \alpha x]_{\alpha} \alpha T(x) \alpha x-x \alpha[T(x), x]_{\alpha} \alpha y \alpha x=0$

Subtracting equation (36) from equation (35) implies,

$$
\begin{aligned}
& x \alpha x \alpha y \alpha[T(x), x]_{\alpha}+3 x \alpha y \alpha x \alpha[T(x), x]_{\alpha}-3 x \alpha y[T(x), x]_{\alpha} \alpha x+2 x \alpha y \alpha[T(x), x]_{\alpha} \alpha x \\
&+[y, x \alpha x]_{\alpha} \alpha x \alpha T(x)-[y, x \alpha x]_{\alpha} \alpha T(x) \alpha x \\
&=x \alpha x \alpha y \alpha[T(x), x]_{\alpha}+3 x \alpha y\left[[x, T(x), x]_{\alpha}\right]_{\alpha}+2 x \alpha y[T(x), x]_{\alpha} \alpha x \\
&+[y, x \alpha x]_{\alpha} \alpha[x, T(x)]_{\alpha}=0
\end{aligned}
$$

which reduces equation (31) to,

$2 x \alpha x \alpha y \alpha[T(x), x]_{\alpha}+3 x \alpha y \alpha x \alpha[T(x), x]_{\alpha}-x \alpha y \alpha[T(x), x]_{\alpha}=0$ for all $x, y \in M$ and $\alpha \in \Gamma$.

By replacing $-[T(x), x]_{\alpha} \alpha x$ by $x \alpha[T(x), x]_{\alpha}$ in the above equation, and by 2 -torsion free $\Gamma$-ring, we get,

$x \alpha x \alpha y \alpha[T(x), x]_{\alpha}+2 x \alpha y \alpha x \alpha[T(x), x]_{\alpha}=0$ for all $x, y \in M$ and $\alpha \in \Gamma$

Recall equation (5) and Lemma 2.5, so, for all $x, y \in M$ and $\alpha \in \Gamma$ we have,

$x \alpha y \alpha[x \alpha x, T(x)]_{\alpha}+2 x \alpha x \alpha y \alpha[T(x), x]_{\alpha}+2 y \alpha x \alpha x \alpha[T(x), x]_{\alpha}+y \alpha x \alpha[x, T(x)]_{\alpha} \alpha x+$

$y \alpha[x, T(x)]_{\alpha} \alpha x \alpha x=0$

Using equation (24) leads to, $x \alpha x \alpha y \alpha[T(x), x]_{\alpha}=0$ for all $x, y \in M$ and $\alpha \in \Gamma$.

Which gives, together with equation (37), $x \alpha y \alpha x \alpha[T(x), x]_{\alpha}=0$

Left multiplication of equation (38) by $T(x)$ gives,

$T(x) \alpha x \alpha y \alpha x \alpha[T(x), x]_{\alpha}=0$ for all $x, y \in M$ and $\alpha \in \Gamma$

Replacing, in equation (38), $T(x) \alpha y$ by $y$ we obtain,

$$
x \alpha T(x) \alpha y \alpha x \alpha[T(x), x]_{\alpha}=0 \text { for all } x, y \in M \text { and } \alpha \in \Gamma
$$

Subtracting (40) from (39) implies, $[T(x), x]_{\alpha} \alpha y \alpha x \alpha[T(x), x]_{\alpha}=0$ for all $x, y \in M$ and $\alpha \in \Gamma$.

Thus, $x \alpha[T(x), x]_{\alpha}=0$ for all $x, y \in M$ and $\alpha \in \Gamma$

In addition, $[T(x), x]_{\alpha} \alpha x=0$ for all $x, y \in M$ and $\alpha \in \Gamma$

Theorem 2.7: Let $M$ be a 2-torsion free semiprime $\Gamma$-ring satisfying condition (A) and $T: M \rightarrow M$ satisfying condition (B), then $T$ is a left and right centralizer.

Proof: We take equation(25), then, for all $x, y \in M$ and $\alpha \in \Gamma$ we get,

$[T(x), x]_{\alpha} \alpha y+x \alpha[T(x), y]_{\alpha}+[T(x), y]_{\alpha} \alpha x+y \alpha[T(x), x]_{\alpha}+x \alpha[T(y), x]_{\alpha}+[T(y), x]_{\alpha} \alpha x=0$.

From equation (37) with the above equation we obtain,

$x \alpha[T(x), y]_{\alpha}+y \alpha[T(x), x]_{\alpha}+x \alpha[T(y), x]_{\alpha}=0$ for all $x, y \in M$ and $\alpha \in \Gamma$.

Left multiplication of the above equation by $[T(x), x]_{\alpha}$, and by equation (38), implies,

$[T(x), x]_{\alpha} \alpha \mathrm{y} \alpha[T(x), x]_{\alpha}=0$ for all $x, y \in M$ and $\alpha \in \Gamma$.

From the semiprime we have, $[T(x), x]_{\alpha}=0$ for all $x \in M$ and $\alpha \in \Gamma$

Combining equation (41) with equation (1) gives $T(x \alpha x)=T(x) \alpha x$ for all $x \in M$ and $\alpha \in \Gamma$.

Also, $T(x \alpha x)=x \alpha T(x)$ for all $x \in M$ and $\alpha \in \Gamma$.

This implies that $T$ is a left and right Jordon centralizer, and by a previous work [8; Theorem 3.1], the result is that $T$ is both a left and right centralizer.

\section{References}

1. Barnes, W. E. 1966. On the $\Gamma$-ring of Nobusawa, Pacific J. Math., 18: 411-422.

2. Luh, J. 1970. A Note on Commuting Automorphism of Rings, Amer. Math. Monthly, 16: 65-75.

3. Kyuno, S. 1978. On Prime Gamma Ring, Pacific J. Math., 75: 185-190.

4. Nobusawa, N. 1964. On the Generalization of the Ring Theory, Osaka J. Math, 1: 81-89.

5. Hoque, Md. F. and Paul, A. C. 2011. On Centralizer of Semiprime Gamma Ring, International Mathematical Forum, 13: 627-638.

6. Shaheen, R. C. 2007. On Centralizer of Some Gamma Ring, Journal Al-Qadisiya for Pure Science, 12(2).

7. Hoque, Md. F. and Paul, A. C. 2013. Centralizer on Semiprime Gamma Ring, Italian J. of Pure and Applied Mathematics, 30: 289-302.

8. Hoque, Md. F. and Paul, A. C. 2011. On Centralizer of Semiprime Gamma Ring, International Mathematical Forum, 6(13): 627-638. 